\title{
High-field transport in semiconductor superlattices for interacting Wannier-Stark levels
}

\author{
Angelo Guida \\ Dipartimento di Fisica Università del Salento, Via Arnesano 73100 Lecce, Italy
}

Lino Reggiani

Dipartimento di Ingegneria dell' Innovazione and CNISM, Universitá del Salento, Via Arnesano s/n, 73100 Lecce, Italy

\author{
Marcello Rosini \\ Dipartimento di Fisica Università di Modena e Reggio Emilia and $\mathrm{S}^{3}$ national \\ research centre of CNR-INFM, Via Campi 213/A, 4100 Modena, Italy \\ E-mail: marcello.rosini@unimore.it
}

\begin{abstract}
We develop a microscopic theory of electron transport in superlattices within the Wannier-Stark approach by including the interaction associated with Zener tuneling among the energy levels pertaining to adjacent quantum wells. By using a Monte Carlo technique we have simulated the hopping motion associated with absorption and emission of polar optical phonons and determined the main transport parameters for the case of a GaAs/GaAlAs structure at room temperature. The interaction among the levels is found to be responsible for a systematic increase of the level energy with respect to the bottom of the quantum well at electric fields above about $20 \mathrm{kV} / \mathrm{cm}$. When compared with the non-interacting case, at the highest fields the average carrier energy evidences a consistent increase which leads to a significant softening of the negative differential value of both the drift velocity and diffusivity.
\end{abstract}

PACS numbers: 05.40.Ca, 02.70.Uu, 68.65.Cd, 73.63.-b, 72.70.+m 


\section{Introduction}

In recent years, superlattices (SLs) received a relevant scientific and technological interest, owing to their nonlinear electrical and optical properties. In particular, at increasing electric field strengths SLs exhibit a nonOhmic behavior with the presence of a negative differential conductivity (NDC) region [1] above a threshold field. In the nonOhmic regime, charge transport can be described in terms of hopping between Wannier-Stark (WS) states [2, 3], thus SLs are good systems for studying hopping transport in semiconductor structures. Despite of the above interest, the knowledge of the transport parameters, like drift velocity and diffusion coefficients for a given SL is still mostly an open problem.

From the analytical side, the problem was investigated with different approaches in Refs. [4, 5, 6, 7, 8]]. The coupling between energy levels and the electric field (the so called Zener effect [9]) was found responsible for the onset of a resonance in the drift velocity [4, 6] and of an antiresonance in the diffusion [6] at fields where both these quantities are decreasing functions of the applied electric field.

From the simulative side, recent Monte Carlo investigations [10, 11] have analyzed the drift velocity [10] and both the drift velocity and diffusion [11] but neglecting the coupling between the field and the energy levels. Furthermore, in Ref. [10]] only general trends are reported so that a quantitive evaluation of the drift velocity is absent.

Here we develop a microscopic theory of electron transport in SLs within the Wannier-Stark approach, which includes the coupling among the energy levels due to the presence of a strong electric field so as to cause Zener tunneling. The theory is applied to an ideally infinite GaAs/GaAlAs superlattice at $300 \mathrm{~K}$. The electric fields considered are in the range $5 \div 200 \mathrm{kV} / \mathrm{cm}$ where the Wannier Stark approach is well justified [12, 13], for which experiments are available [14]. A comparative investigation of the effect of the interaction among energy levels with respect to the case of independent energy levels is then carried out.

The content of the paper is organized as follows. In Sec. 2 we define the physical system under investigation and present the theoretical approach to be used within a Monte Carlo simulator. In Sec. 3 the main results concerning the drift velocity and the diffusion coefficient are presented. Major conclusions are drawn in Sec. 4.

\section{Theoretical approach}

\subsection{Theory of WS states}

We consider a SL made by alternated layers of different semiconductors (A and B) along the $z$-direction. The single electron Schrödinger equation in the presence of an external electric field along the $z$-direction $\mathbf{F}=(0,0, F)$ is:

$$
\left[\frac{\hat{\mathbf{p}}^{2}}{2 m^{*}}+V_{S L}(\mathbf{r})-e F z\right] \psi(\mathbf{r})=\varepsilon \psi(\mathbf{r})
$$


High-field transport in semiconductor superlattices for interacting Wannier-Stark levels3

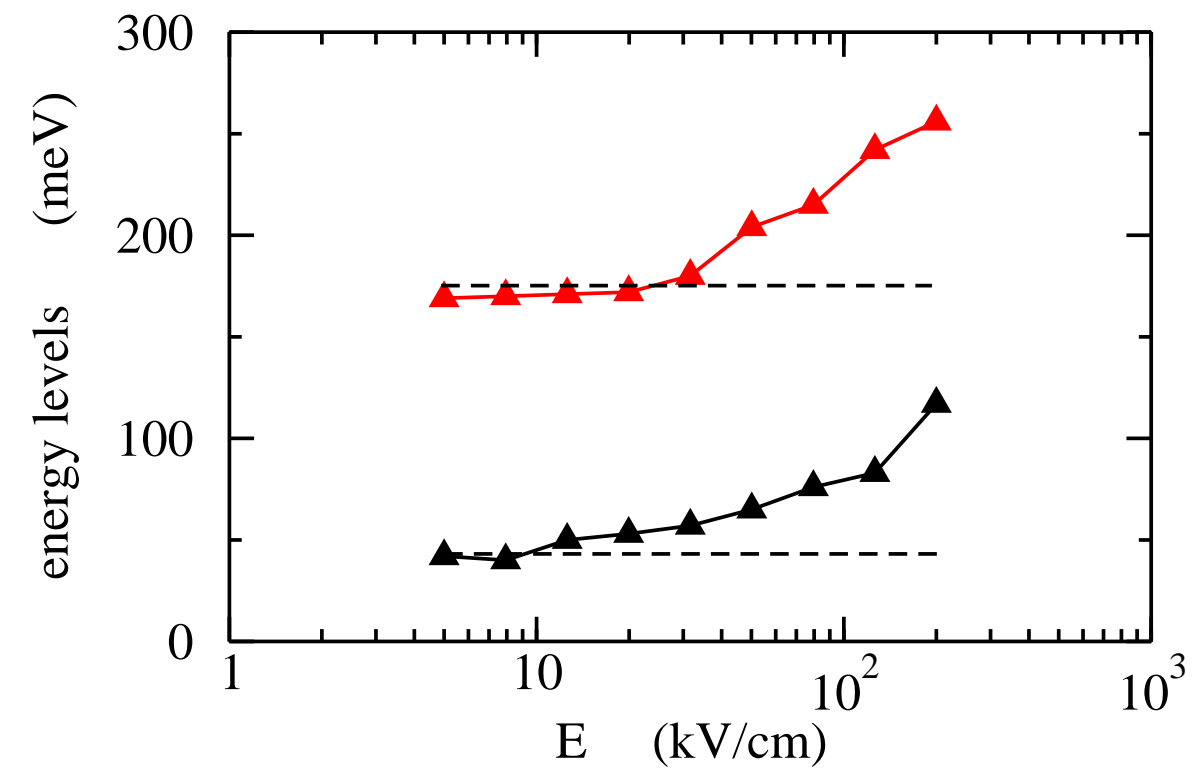

Figure 1. Eigenvalues of the first and second energy levels of a quantum well pertaining to an ideal infinite SL. Symbols refer to the case of interacting energy levels, dashed lines to the case of independent energy levels.

where $\hat{\mathbf{p}}$ is the momentum operator, $m^{*}$ is the effective mass assumed to be the same in the well and in the barrier, $V_{S L}(\mathbf{r})$ is the potential which determines the barrier height of the SL, and $\psi(\mathbf{r})=\varphi(z) g(x, y)$ with $g$ the Bloch function. The SL potential is taken of the Kronig-Penney form:

$$
V_{S L}(z)= \begin{cases}V_{0} & \text { if } 0<z<a \\ 0 & \text { if } a<z<a+b\end{cases}
$$

with $a$ and $b$ the length of the well and of the barrier, respectively, and $d=a+b$ the SL period. Accordingly, using the Dirac notation the Schrödinger equation in the $z$-direction is:

$$
\left[\widehat{H}_{0}-e F z\right]|\varphi\rangle=\epsilon|\varphi\rangle \text {. }
$$

The solution of Eq. (3) can be expanded over the SL Bloch states $\left|n, k_{z}\right\rangle$, with $n$ the band index, which are eigenstates of the Hamiltonian $\widehat{H}_{0}$ of the SL in the absence of the electric field

$$
|\varphi\rangle=\sum_{n^{\prime}} \int_{k_{z}^{\prime}} \mathrm{d} k_{z}^{\prime}\left\langle k_{z}^{\prime} n^{\prime} \mid \varphi\right\rangle\left|k_{z}^{\prime} n^{\prime}\right\rangle
$$

and substituted into the above Schrödinger equation; multiplying by $\left\langle k_{z} n\right|$ we develop the calculation and obtain

$$
\begin{aligned}
& {\left[E_{n}\left(k_{z}\right)-\epsilon\right]\left\langle k_{z} n \mid \varphi\right\rangle-} \\
& e F \sum_{n^{\prime}} \int_{k_{z}^{\prime}} \mathrm{d} k_{z}^{\prime}\left\langle k_{z} n|z| k_{z}^{\prime} n^{\prime}\right\rangle\left\langle k_{z}^{\prime} n^{\prime} \mid \varphi\right\rangle=0 .
\end{aligned}
$$


High-field transport in semiconductor superlattices for interacting Wannier-Stark levels4
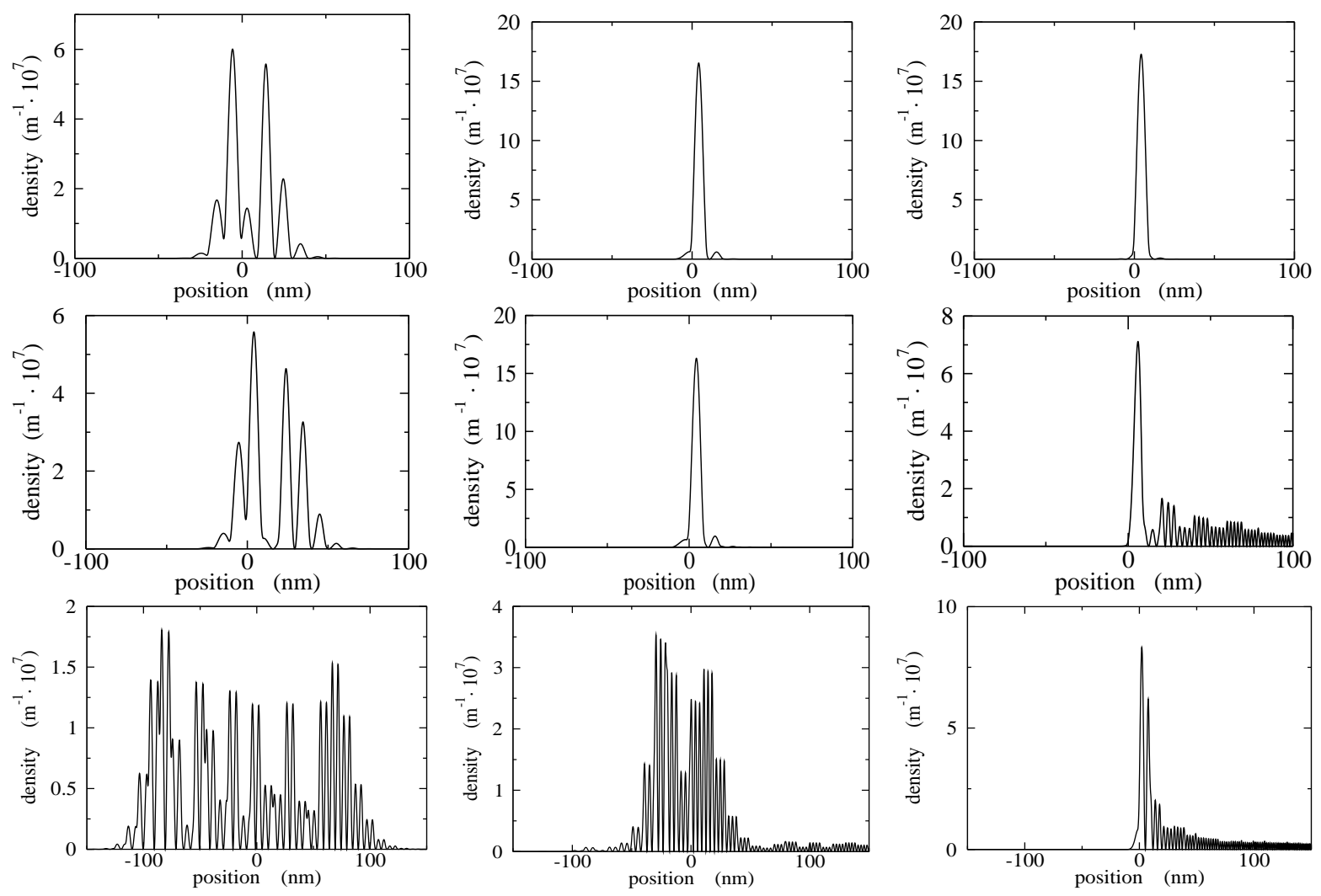

Figure 2. Normalized square value of the Wannier Stark wavefunction at different electric field strengths of, respectively from right to left, 5,30 and $200 \mathrm{kV} / \mathrm{cm}$. The first raw of figures refers to the first level in the absence of interaction; the second raw of figures refers to the first level in the presence of interaction; the third raw of figures refers to the second level in the presence of interaction.

Here, the value $\left\langle k_{z} n|z| k_{z}^{\prime} n^{\prime}\right\rangle$ is calculated explicitly and, after some algebra, it results to be

$$
\left\langle k_{z} n|z| k_{z}^{\prime} n^{\prime}\right\rangle=\frac{1}{i} \frac{\mathrm{d}}{\mathrm{d} k_{z}^{\prime}} \delta_{n n^{\prime}} \delta\left(k_{z}-k_{z}^{\prime}\right)+X_{n n^{\prime}} \delta\left(k_{z}-k_{z}^{\prime}\right)
$$

with

$$
X_{n n^{\prime}}=\frac{2 \pi i}{L} \int \mathrm{d} z u_{n k_{z}}^{*}(z) \frac{\mathrm{d}}{\mathrm{d} k_{z}^{\prime}} u_{n^{\prime} k_{z}^{\prime}}(z) .
$$

where $u_{n k_{z}}$ is the periodic part of the Bloch function. The above matrix elements include the interaction among levels pertaining to different quantum wells caused by the electric field and they are basically related to Zener interband tunneling. We recall, that under the conditions of moderate electric fields and weak coupling between adjacent wells it is justified to consider a one-band model for the calculation, thus neglecting off-diagonal matrix elements described by eq. (7). This approximation leads to a simple solution of eq. (5) and was already applied in Ref. [[10, 11]] where the WS eigenstates have been obtained analytically [11]. 


\subsection{Numerical method}

In the present paper we point to fully take into account the off-diagonal terms (7) in the calculation of the WS eigenstates and further of the SL transport properties. However, in this case it is not possible to get a simple analytical solution of the system. Thus, to accomplish this purpose, we have decided to solve numerically the Schrödinger equation along the $z$ direction (3). With this approach, no interaction is dropped during the calcualtion and the interaction decribed by (7) is automatically included in the solution. Looking for a solution of the Schrödinger equation at the finite differences, we consider a number $M$ of cells (typically 30 cells in the backward direction and 30 in the forward direction for a total number of $M=61$ ) representative of an ideal infinite SL. (Indeed, electron wavefunctions are found to extend up to a maximum of 20 cells). Then, the Schrödinger equation is discretized in a number of $N$ points (typically $N=100$ ) equally spaced inside a single cell, $M \times N$ being the total number of points used to obtain the numerical solution within an estimated convergence of a few percent. Accordingly, $z \rightarrow i \Delta, \varphi(z) \rightarrow \varphi_{i}$, with $\Delta=\frac{d}{N}$, and defining

$$
\begin{gathered}
d_{i}=\frac{\hbar^{2}}{m^{*}} \frac{1}{\Delta^{2}}+V_{i}-e F i \Delta \\
\mathbf{A}=\left(\begin{array}{cccccc}
d_{1} & e & 0 & \ldots & \ldots & 0 \\
e & d_{2} & e & 0 & \ldots & 0 \\
0 & e & d_{3} & e & \ldots & 0 \\
\vdots & & \ddots & \ddots & \ddots & \vdots \\
0 & \ldots & 0 & e & d_{N M-1} & e \\
0 & \ldots & \ldots & \ldots & e & d_{N M}
\end{array}\right)
\end{gathered}
$$

the discretized Schrödinger equation of the system takes the compact form:

$$
\mathbf{A} \varphi=\varepsilon \varphi
$$

with $\varphi=\left(\varphi_{1}, \varphi_{2}, \ldots, \varphi_{N M}\right)$.

Since the matrix $\mathbf{A}$ is real and symmetric the eigenvalues are real, distinguishable, and in number equal to the order of the matrix $M \times N$. The eigenvectors $\varphi^{i}$ are the Wannier-Stark wavefunctions. In the present case, the $\varphi_{j}^{i}$ are two-dimensional vectors with the index $i$ representing the corresponding energy level and the index $j$ the pertaining grid point. The eigenvalue $\varepsilon^{i}$ and the eigenvector $\varphi^{i}$, being labelled with the same index $i$, are in full correspondence.

The eigenvalues are then classified according to the pertaining quantum well and energy level by using the properties of the Wannier-Stark eigenvectors (for a review of the WS functions properties see for example [15]). In particular we know that WS functions should be localized and satisfy the translational symmetry $\varphi_{n+m}(z)=\varphi_{n}(z-m d)$ for any pairs of quantum wells labelled by $(n, m)$. Then, as validity test of the 
numerical algorithm, the energy difference between adjacent wells is calculated and their ladder distribution verified. We have also verified that in the central region of the representative device, where boundary effects are negligible, the translational property of the eigenvectors belonging to the same energy level is accomplished. In this way, for each value of the electric field we select the wavefunction representative of the infinite SL.

\subsection{Electron-phonon interaction}

The calculation of the polar-optical phonon scattering probability per unit time in the WS representation follows the standard Fermi Golden Rule. In brief, after decoupling the phonon bath, the point-to-point transition probability per unit time is

$$
\begin{aligned}
& P\left(\nu n \mathbf{k}_{\|}, \nu^{\prime} n^{\prime} \mathbf{k}_{\|}^{\prime}\right)=\frac{2 \pi}{\hbar} \sum_{\mathbf{q}} c^{2}(\mathbf{q})\left[\begin{array}{c}
n_{\mathbf{q}} \\
n_{\mathbf{q}}+1
\end{array}\right] \times \\
& \left|\left\langle\nu^{\prime} n^{\prime}\left|e^{\mp i q_{z} z}\right| \nu n\right\rangle\right|^{2} \times \\
& \delta_{\mathbf{k}_{\|}-\mathbf{k}_{\|}^{\prime} \mp \mathbf{q}_{\|}} \delta\left[\epsilon\left(n \mathbf{k}_{\|}\right)-\epsilon\left(n^{\prime} \mathbf{k}_{\|}^{\prime}\right) \mp \hbar \omega_{o p}+\nu e F d\right]
\end{aligned}
$$

where $n_{\mathbf{q}}$ is the mean value of the phonon occupation number, $\mathbf{q}=\left(\mathbf{q}_{\|}, q_{z}\right)$ is the phonon wave vector and $\mathbf{k}=\left(\mathbf{k}_{\|}, k_{z}\right)$. For $c^{2}(\mathbf{q})$ we take the standard Frölich squared matrix element [16]:

$$
c^{2}(\mathbf{q})=\frac{\hbar e^{2} \omega_{o p}}{2 V \epsilon_{0}}\left(\frac{1}{\epsilon_{+}}-\frac{1}{\epsilon_{-}}\right) \frac{\mathbf{q}^{2}}{\left(\mathbf{q}^{2}-\mathbf{q}_{D}^{2}\right)^{2}}
$$

where $\omega_{o p}$ is the optical phonon frequency, $\epsilon_{-}, \epsilon_{+}$are the static and high frequency relative dielectric constant, $\mathbf{q}_{D}$ is the screening wave vector and $V$ is the crystal volume.

Being interested in the total scattering probability per unit time from an initial state $\left(\nu n \mathbf{k}_{\|}\right)$to any possible final state $\mathbf{k}_{\|}^{\prime}$, we integrate over the final momentum variable and obtain the integrated transition probability per unit time:

$$
\begin{gathered}
P_{\nu^{\prime} n^{\prime}}\left(\nu n \mathbf{k}_{\|}\right)=\frac{m^{*}}{(2 \pi)^{5} \hbar^{3}}\left[\begin{array}{c}
n \\
n+1
\end{array}\right] \times \\
\int \mathrm{d} \theta \int \mathrm{d} q_{z} c^{2}(\mathbf{q})\left|\left\langle\nu^{\prime} n^{\prime}\left|e^{\mp i q_{z} z}\right| \nu n\right\rangle\right|^{2} .
\end{gathered}
$$

Having integrated the $\delta$ function in Eq. (9) implies having accounted for momentum conservation that leads to the constraint $\mathbf{q}=\left(k_{x}^{\prime}-k_{x}, k_{y}^{\prime}-k_{y}, q_{z}\right)$. This last Eq. (11) is not further integrable with standard method because of the not simple dependence upon q. Within the Monte Carlo technique this step is accomplished using the rejection technique [17].

Equation (11) describes the probability that an electron with initial state $\left(\nu n \mathbf{k}_{\|}\right)$ is scattered to a final state in well $\nu^{\prime}$, in band $n^{\prime}$, with any $\mathbf{k}_{\|}^{\prime}$. However, in our approach electrons are allowed to jump a maximum distance of 6 wells forward and 6 wells backward, since the hopping transition element is negligible for longer distances. Indeed, we have verified that, for the transport parameters investigated here, the results 
are not affected by a further increase of the maximum hopping distance. Accordingly, the associated transition probabilities per unit time for a carrier jump from an initial well to any of the 12 final wells due to absorption and emission of polar optical phonons is evaluated and used inside a Monte Carlo procedure, as detailed in Ref. [[11]]. For sake of simplicity, other scattering mechanisms are neglected and we have considered at most only the first two energy levels in each well. The time evolution of the carrier ensemble is then evaluated for a period sufficiently long to extract the drift velocity and the longitudinal diffusion coefficient from the corresponding slopes of the average value of the position and of its variance along the field direction [11].

\section{Results and discussion}

The theory is applied to the case of a GaAs/GaAlAs SL at $300 \mathrm{~K}$, being that the most studied SL. As typical parameters we take $a=8.5 \mathrm{~nm}, b=1.5 \mathrm{~nm}$ and a barrier height of $0.4 \mathrm{eV}$. For the electron effective mass we take everywhere the value of GaAs $m^{*}=0.067 m_{0}$, with $m_{0}$ the free electron mass, for the polar optical phonon energy we take $\varepsilon_{o p}=35 \mathrm{meV}$, and for the polar optical coupling we take $\frac{1}{\epsilon_{+}}-\frac{1}{\epsilon_{-}}=0.013$. The electric fields considered are in the range $5 \div 200 \mathrm{kV} / \mathrm{cm}$ where the Wannier Stark approach is well justified [13].

An ensemble Monte Carlo simulation has been set-up in order to simulate electron transport transport in this superlattice, as detailed in [[11]] and the estimated physical quantities are calculated as follows

$$
\begin{aligned}
\left\langle v_{z}\right\rangle & =\frac{1}{N \delta t} \sum_{i=1}^{N} \delta z_{i} \\
D_{z} & =\frac{1}{2} \frac{\delta\left\langle(\Delta z)^{2}\right\rangle}{\delta t} \\
\langle\epsilon\rangle & =\frac{1}{N} \sum_{i=1}^{N} \epsilon_{i}
\end{aligned}
$$

for drift velocity, diffusion coefficient end mean energy respectively, where $z_{i}$ and $\epsilon_{i}$ are the position and the energy of the $i$-th particle.

The results of the investigation are reported in Figs. 1 to 5 . Here, the case of interacting levels (through Zener tunneling) is compared to the case of independent levels at increasing values of the electric field.

Figure 1 reports the energy of the first and second level inside the quantum well (measured from the bottom of the well) as function of the applied field. In the absence of interaction the energy of the first and second levels are found to be of 43 and $175 \mathrm{meV}$, respectively. The presence of the interaction is responsible for a significant increase, up to about a factor two, of the energy levels at fields starting from about $10 \mathrm{kV} / \mathrm{cm}$. Such an increase is associated with the broadening of energy levels [18, 19].

Figure 2 reports the square modulus of the Wannier Stark wavefunctions associated with the first and second energy level at increasing electric fields (from left to right). The 
High-field transport in semiconductor superlattices for interacting Wannier-Stark levels8

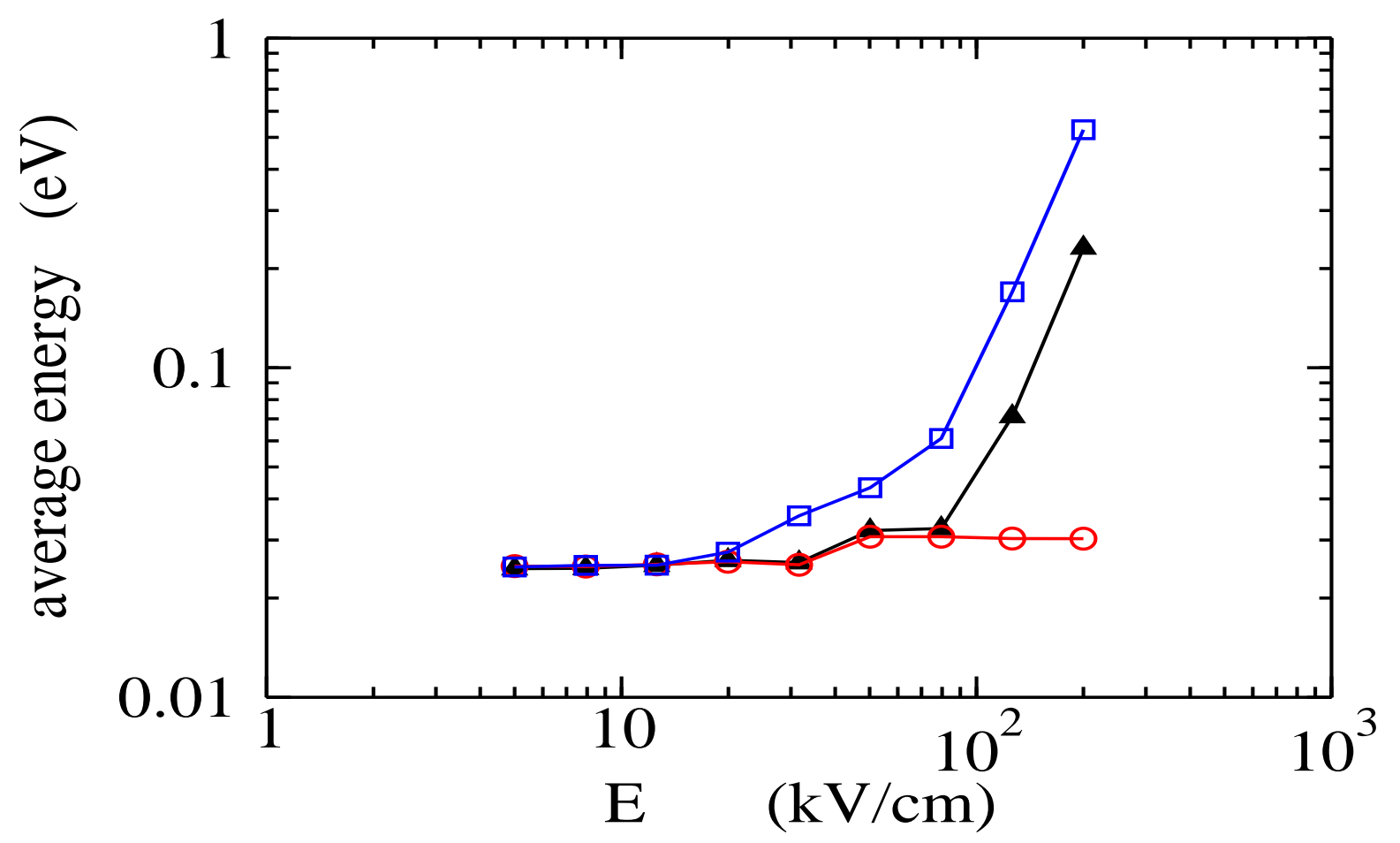

Figure 3. Average carrier energy as function of the applied electric field. Open circles, full triangles, and open squares refer to the cases when only the first level in the absence of interaction, only the first level in the presence of interaction, both the first and second level in the presence of interaction are considered. Curves are guides to eyes.

set in the first (second) raw of the figure refers to independent (interacting) first energy levels. The tendency to localization exhibited by both cases at moderately increasing fields is found to be broken at the highest fields by the presence of the coupling between level and field, as evidenced by the long tail in the direction of the field exhibited by the wavefunction in the second raw. This tail is originated by the coupling of different bands of the superlattice induced by the electric field, as described in Eq. (77), and enables us to evaluate the effect of carrier tunneling from a well to those in the forward direction (Zener effect [9]). The agreement between analytical and numerical results at moderate electric fields is taken as a validation test of the numerical algorithm used here. The set in the third raw of Fig. 2 refers to the case of interacting second energy levels. Here, at low fields the delocalization is stronger than in the case of the first level, as expected for a higher energy level. At increasing fields we observe an increase of localization and the appearance of the long tail in the direction of the field.

Figure 3 reports the carrier average energy obtained from Monte Carlo simulations at increasing electric fields. Above $50 \mathrm{kV} / \mathrm{cm}$, the presence of interacting energy levels is found to be responsible for a substantial increase of the average energy (up to about a factor of 10) associated with the increased spatial mobility of the carriers due to the Zener effect. The inclusion of the second energy level is found to emphasize the trend of energy increase.. 
High-field transport in semiconductor superlattices for interacting Wannier-Stark levels9

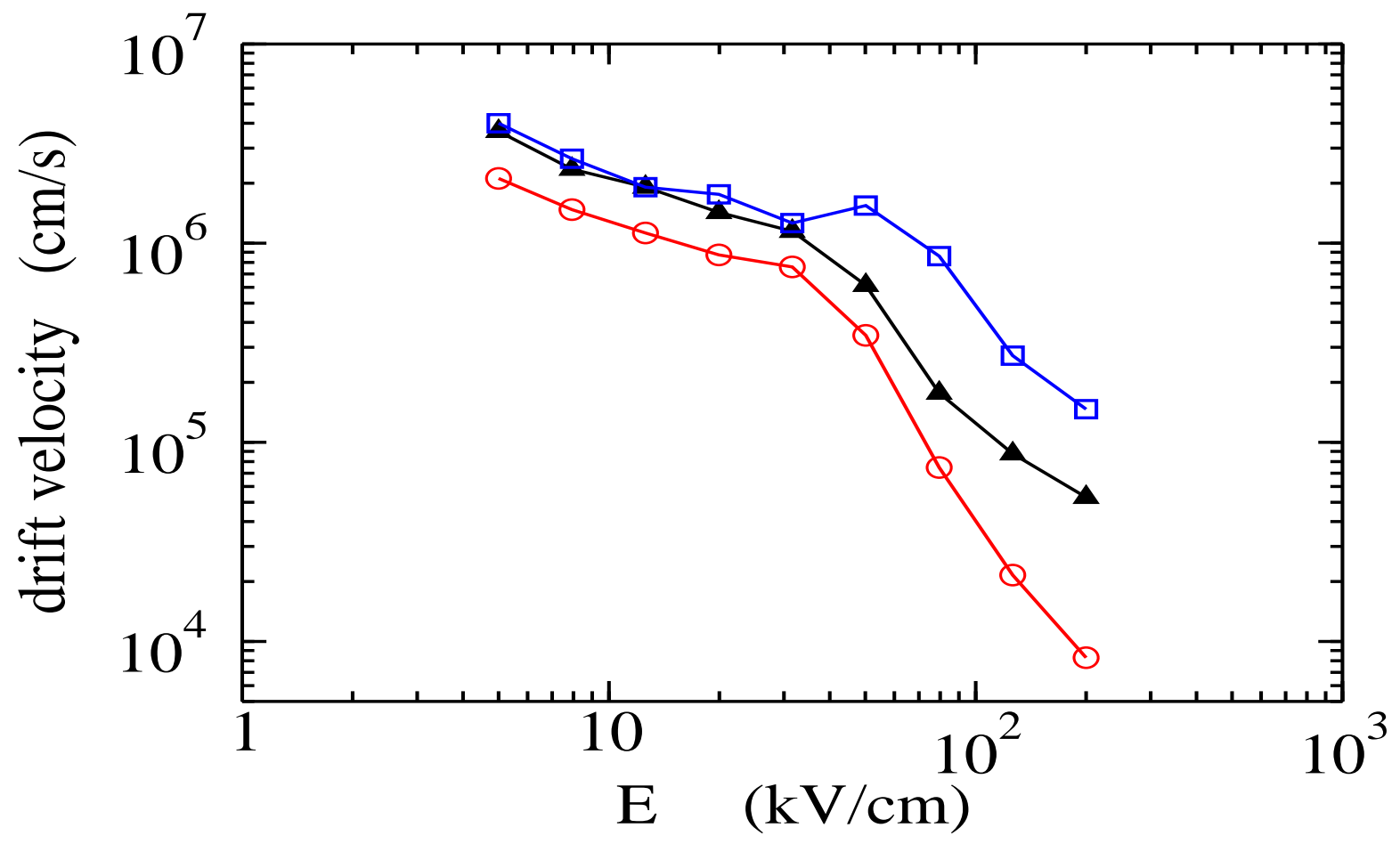

Figure 4. Average carrier velocity as function of applied electric field. Full triangles, open circles and open squares refer to the cases when only the first level in the absence of interaction, only the first level in the presence of interaction, both the first and second level in the presence of interaction are considered. Curves are guides to eyes.

Figures 4 and 5 report the average velocity and the longitudinal diffusion coefficient at increasing fields. Apart from a numerical off-set within a factor of two, in the region up to about $50 \mathrm{kV} / \mathrm{cm}$, both quantities evidence the same systematic decrease with increasing fields. Such a decrease is associated with the localization trend of the wavefunction [20] and it is called negative differential mobility (NDM). Above $50 \mathrm{kV} / \mathrm{cm}$, the simulations show a substantial increase (up to about a factor of 10) of both drift and diffusion with respect to the independent case, in concomitance with that of the average energy. This softening of the negative diferential behaviour of both drift and diffusion is associated with the onset of the Zener effect. The inclusion of the second energy level is found to emphasize the trend to increase of both the kinetic coefficients. We remark that simulations evidence an appreciable kink of both drift and diffusion at a field around $40 \mathrm{kV} / \mathrm{cm}$, which is associated with the resonance occurring when the potential energy due to the voltage drop on a period equals the optical phonon energy [4, 11].

For a microscopic interpretation of the above results, we refer to the shape of the total hopping rate from an initial well $\nu$ to other final wells $\nu^{\prime}$ for a forward or backward hop in the simple case of a single level, $P_{\nu \nu^{\prime}}$. The maximum value of these rates, which are used in Monte Carlo simulations to generate a hop between states, are reported in Figs. 6 to 8 at different field strengths for the case of independent and interacting first energy levels.

Figure 6 shows that at low fields the independent and interacting models give 


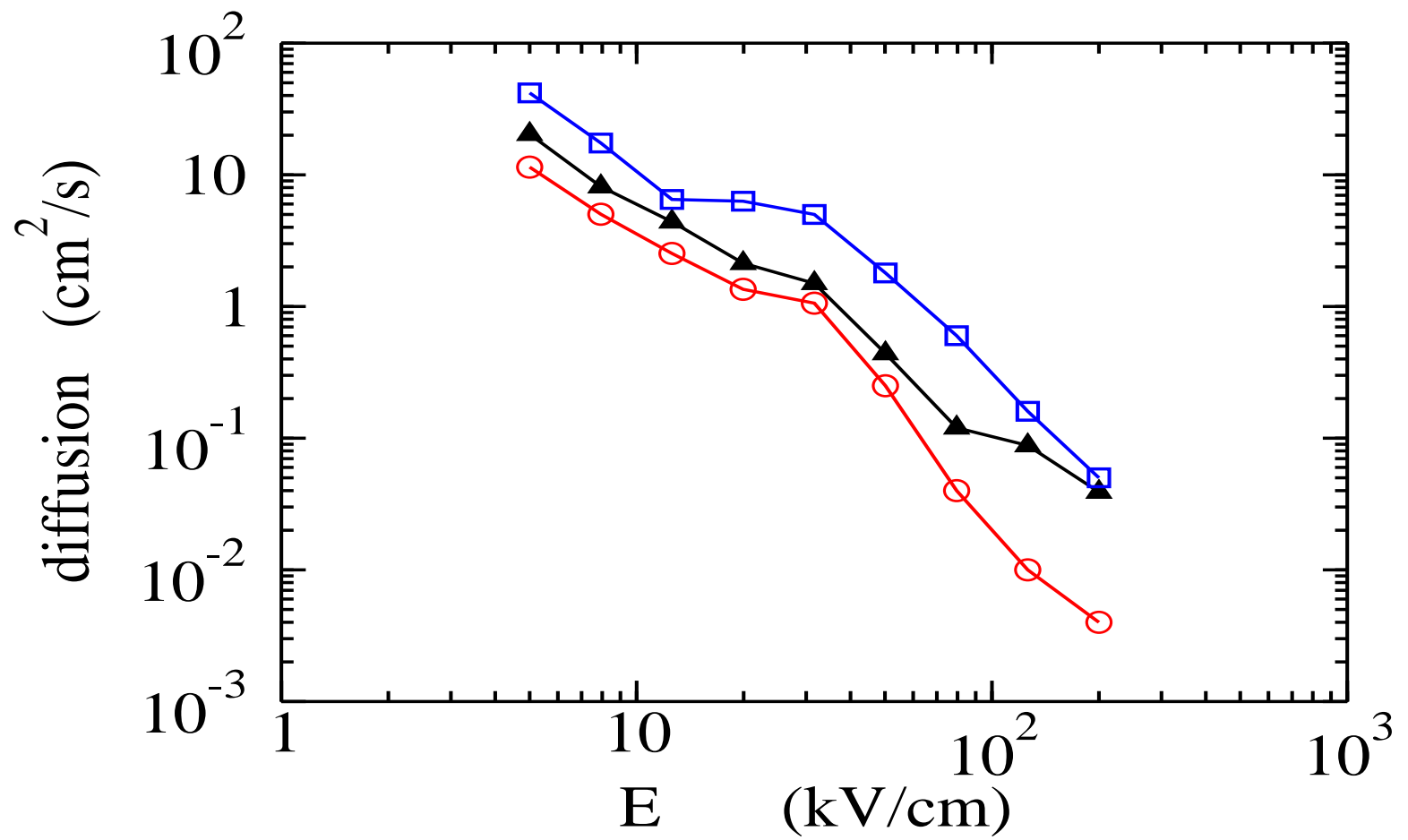

Figure 5. Carrier longitudinal diffusion coefficient as function of the applied electric field. Full triangles, open circles and open squares refer to the cases when only the first level in the absence of interaction, only the first level in the presence of interaction, both the first and second level in the presence of interaction are considered. Curves are guides to eyes.

practically the same results (we notice that the forward value is always greater than the corresponding bacward value, thus ensuring a net motion of the carrier ensemble along the field direction). Hopping rates at increasing distances decreases exponentially as expected by the localized feature of the electron wavefunction. Figures 7 and 8 show that the presence of the interaction leads to a systematic increase of the rates expecially of those to far neighbour wells. Accordingly, the drift and diffusion in the presence of interacting levels increases with respect to the case of independent levels.

The general behaviour of the drift velocity is in good qualitative agreement with the analytical results of Ref. [4]] in the common range of fields. Furthermore, recent experiments [14] on the drift velocity in GaAs/GaAlAs SLs evidence a saturation behavior of the drift velocity for electric fields in the range $20 \div 50 \mathrm{kV} / \mathrm{cm}$, which is in qualitative agreement with present results. In this respect, we notice that for a proper experimental validation of the theory more quantitative experiments are necessary. In this context, we remark that present results on the diffusion coefficient do not evidence the Zener antiresonance predicted by analytical calculations [6]. The lack of a sufficient resolution of the results due to the numerical uncertainty, estimated to be within a factor 2 at worst, and/or the presence of a substantial carrier heating at the highest fields, could be the reasons for the missed evidence of the antiresonance in the diffusion coefficient. 


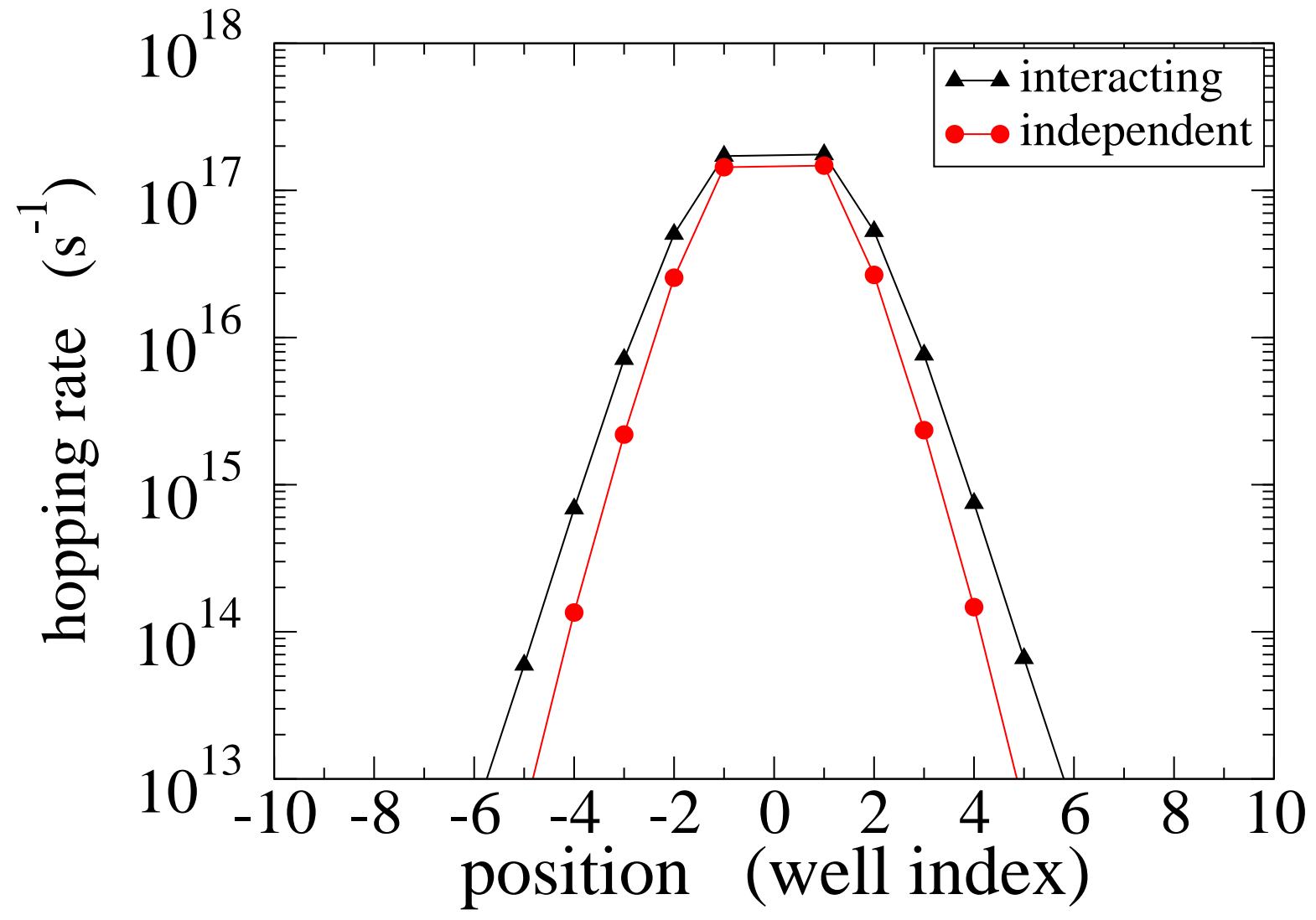

Figure 6. Maximum hopping rate from a given inital well $\nu=0$ to a final well in the forward (positive) and bacward (negative) direction. Calculations are performed for the case of a single level and $E=13 \mathrm{kV} / \mathrm{cm}$. Full circles refer to independent levels, full triangles to interacting levels. Curves are guides to eyes.

\section{Conclusions}

We have investigated transport properties of an ideal infinite superlattice in the presence of interacting energy levels associated with the presence of an applied electric field. To this purpose the one dimensional Schrödinger equation in the direction of the applied field has been numerically solved and an ensemble Monte Carlo technique used to simulate the carrier motion in the three-dimensional states of a perfect superlattice. Theory is applied to a GaAs/GaAlAs structure with a period of $10 \mathrm{~nm}$ at room temperature. The main results of the present work can be summarized as follows. (i) the interaction among the levels is found to be responsible for a systematic increase of the energy levels with respect to the bottom of the quantum well. (ii) the same interaction is found to be responsible for the onset of a new delocalization of the Wannier-Stark states at the highest fields associated with the tunneling of electrons between adjacent quantum wells. (iii) with respect to the non-interacting model, for fields above about $20 \mathrm{kV} / \mathrm{cm}$ the average carrier energy as well as the drift velocity and the longitudinal diffusion coefficient evidence a consistent increase (up to about a factor of 10). Such an increase leads to a significant softening of the negative differential value of both the 


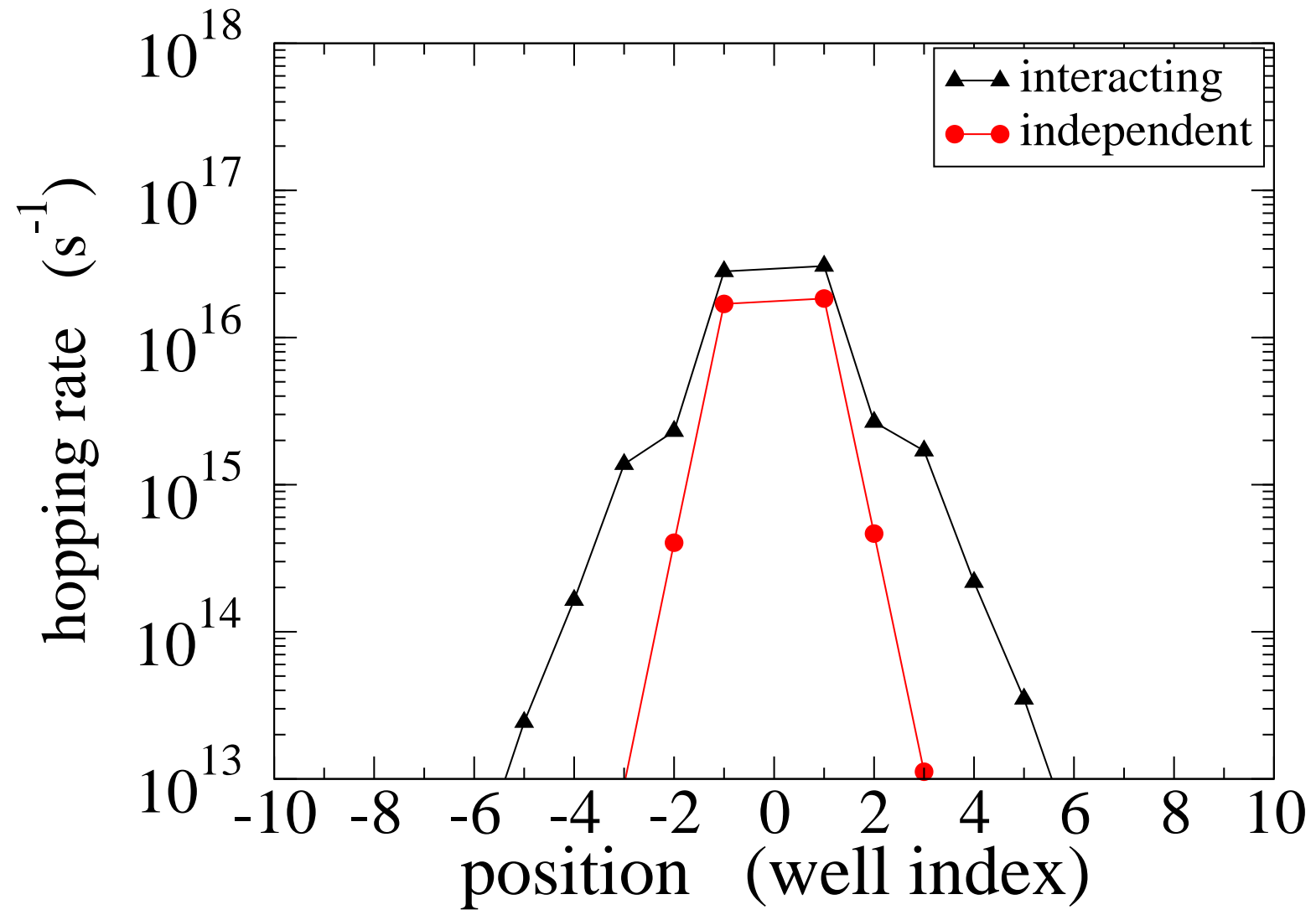

Figure 7. Maximum hopping rate from a given inital well $\nu=0$ to a final well in the forward (positive) and bacward (negative) direction. Calculations are performed for the case of a single level and $E=50 \mathrm{kV} / \mathrm{cm}$. Full circles refer to independent levels, full triangles to interacting levels. Curves are guides to eyes.

drift velocity and diffusivity at fields where Bloch oscillation regime should be active.

[1] H.T. Grahn, K. von Klitzing, K. Ploog, and G.H. Dohler, Phys. Rev. B43, 12094 (1991).

[2] G. H. Wannier, Rev. Mod. Phys 34, 645 (1962)

[3] A. Wacker and A.-P. Jauho, Phys. Rev. Lett. 80, 369 (1998).

[4] S. Rott, N. Linder and G.H. Döhler, Phys. Rev. B65, 195301 (2002).

[5] V.V. Bryksin and P. Kleinert, J. Phys.: Condens. Matter 15, 1415 (2003).

[6] P. Kleinert and V. V. Bryksin, J. Phys.: Condens. Matter 16, 4441 (2004).

[7] L. G. Mourokh, A. Y. Smirnov, and N. J. M. Horing, Phys. Lett. A 269, 175 (2000)

[8] L. L. Bonilla and R. Escobedo, and A. Perales, Phys. Rev. B 68, 241304(R) (2003)

[9] C. Zener, Proc. R. Soc. London, Ser. A 145, 523 (1934)

[10] Yu. A. Tarakanov, V. Vettchinkina, M.A. Odnoblyudov, K.A. Chao, N. Sekine and K. Hirakawa, Phys. Rev. B72, 125345 (2005).

[11] M. Rosini and L. Reggiani, Phys. Rev. B72, 195304 (2005).

[12] A. Wacker, A.-P. Jauho, S. Rott, A. Markus, P. Binder and G. H. Dohler, Phys. Rev. Lett. 83, $836(1999)$

[13] A. Wacker, and A.-P. Jauho, Phys. Rev. Lett. 80, 369 (1998).

[14] S. Madhavi, M. Abe, Y. Shimada, and K. Hirakawa, Phys. Rev. B65, 193308 (2002).

[15] A. Wacker, Phys. Rep. 357, 1 (2002).

[16] H. Haken, Quantum field theory of solids - AN introduction (North Hollan, Amsterdam), 1976

[17] C.Jacoboni and L.Reggiani Rev. Mod. Phys. 55, 645 (1983). 


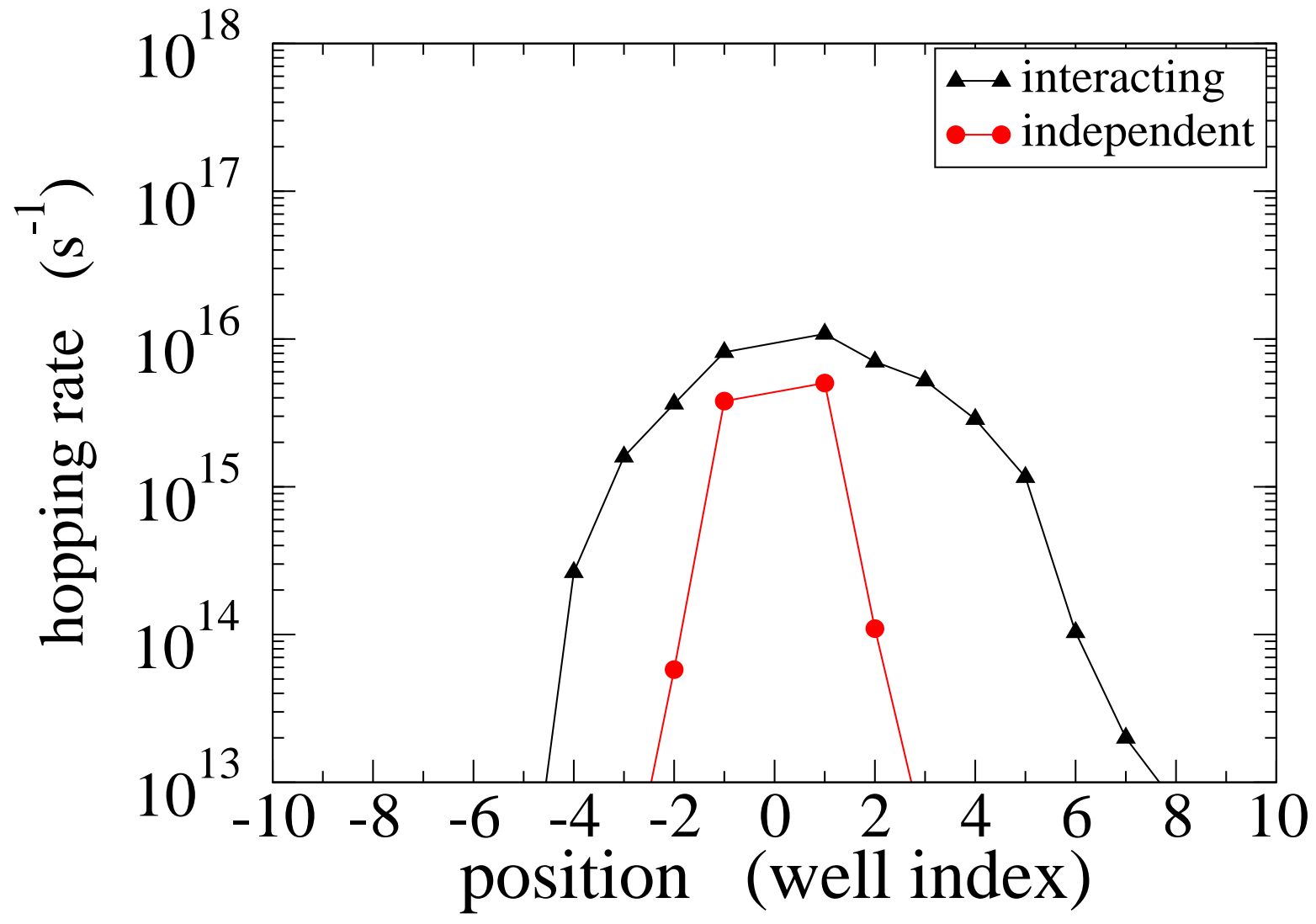

Figure 8. Maximum hopping rate from a given inital well $\nu=0$ to a final well in the forward (positive) and bacward (negative) direction. Calculations are performed for the case of a single level and $E=200 \mathrm{kV} / \mathrm{cm}$. Full circles refer to independent levels, full triangles to interacting levels. Curves are guides to eyes.

[18] M. Nakayama, I. Tanaka, H. Nishimura, K. Kawashima and K. Fujiwara Phys. Rev. B44, R5935 (1991).

[19] S. Glutsch, Phys. Rev. B69, 235317 (2004).

[20] L. Esaki, and R. Tsu, IBM J. Res. Dev., 1461 (1970). 